\title{
A CONVERGENCE EQUIVALENCE RELATED TO POLYNOMIALS ORTHOGONAL ON THE UNIT CIRCLE
}

\author{
BY \\ GLEN BAXTER(')
}

1. Introduction. We are concerned here with a conjecture made by the author in a previous paper [1]. We prove this conjecture for real-valued functions and demonstrate its use in the theory of Toeplitz determinants and in harmonic analysis.

Let $f(\theta)$ be an integrable function on $-\pi \leqq \theta \leqq \pi$ with Fourier coefficients $c_{k}$, i.e.,

$$
f(\theta) \sim \sum_{k=-\infty}^{\infty} c_{k} e^{i k \theta}
$$

and for every $n \geqq 0$ let

$$
D_{n}(f)=\left|\begin{array}{cccc}
c_{0} & c_{-1} & \cdots & c_{-n} \\
c_{1} & c_{0} & \cdots & c_{-n+1} \\
\vdots & \vdots & \ddots & \vdots \\
c_{n} & c_{n-1} & \cdots & c_{0}
\end{array}\right| .
$$

Finally, if $D_{n}(f) \neq 0$ for $n \geqq 0$, let for $n \geqq 1$

$$
\alpha_{n}=(-1)^{n} \frac{D_{n-1}\left(e^{-i \theta} f\right)}{D_{n-1}(f)},
$$

and

$$
\beta_{n}=(-1)^{n} \frac{D_{n-1}\left(e^{i \theta} f\right)}{D_{n-1}(f)} .
$$

We now state the conjecture.

Conjecture. Let $f(\theta)$ be a bounded, measurable function on $-\pi \leqq \theta \leqq \pi$ and let $D_{n}(f) \neq 0$ for all $n \geqq 0$. Then, $\log f(\theta)$ is integrable and has an absolutely convergent Fourier series if, and only if, $\sum\left|\alpha_{n}\right|<\infty$ and $\sum\left|\beta_{n}\right|<\infty$.

Presented to the Society, December 27, 1960 under the title On the asymptotic behavior of Toeplitz determinants, and January 9, 1961 under the title On the asymptotic behavior of Toeplitz determinants. II; received by the editors January 23, 1961.

(1) This work was supported by the United States Air Force through the Air Force Office of Scientific Research of the Air Research and Development Command, under contract No. AF AFOSR-61-4. Reproduction in whole or in part is permitted for any purpose of the United States Government. 
If $f(\theta)$ is real-valued, then $c_{-k}=\bar{c}_{k}$ and $\beta_{n}=\bar{\alpha}_{n}$. We consider only this case of the conjecture in this paper. However, our proof is more general than this special case in two ways. In the first place, two steps of our three step proof are quite general. Only the third step of $\$ 7$ seems missing from the proof of the general conjecture. In the second place, the methods show a much stronger connection between the convergence properties of $\left\{\alpha_{n}\right\}$ and $\left\{\beta_{n}\right\}$ and the Fourier series of $\log f(\theta)$ than is stated above. Let $\nu(n) \geqq 1$ be an even function of the integer $n$ such that $\nu(n) \leqq \nu(m) \nu(n-m)$ for every $n, m$ and such that $\nu(n) / n^{\lambda} \rightarrow 0$ for some $\lambda \geqq 0$. In the notation of (1.1) let

$$
\|f\|_{\nu}=\sum_{n=-\infty}^{\infty} \nu(n)\left|c_{n}\right|,
$$

and set

$$
\|\alpha\|_{\nu}=\sum_{n=1}^{\infty} \nu(n)\left|\alpha_{n}\right|
$$

Then, our main theorem is the following:

THEOREM 1.1. Let $f(\theta)$ be a real, bounded, measurable function on $-\pi \leqq \theta \leqq \pi$ and let $D_{n}(f) \neq 0$ for all $n$. Then, $\log f(\theta)$ is integrable and $\|\log f\|_{\nu}$ is finite if, and only if, $\|\alpha\|_{\nu}$ is finite.

According to the Wiener-Lévy Theorem (see §2.c) if $f(\theta)$ is a positive continuous function, then $\|f\|_{\nu}$ is finite if, and only if, $\|\log f\|_{\nu}$ is finite. Moreover, $D_{n}(f)>0$ if $f(\theta)$ is positive. We can thus state an important corollary to Theorem 1.1.

Corollary 1.1. Let $f(\theta)$ be a positive, continuous function on $-\pi \leqq \theta \leqq \pi$. Then, $\|f\|_{\nu}$ is finite if, and only if, $\|\alpha\|_{\nu}$ is finite.

We turn now to a brief discussion of possible applications of Theorem 1.1.

2. Applications. For convenience we consider in this section only the case $f(\theta)>0$. Szegö $[3$, Chapter II $]$ has defined a set of polynomials $\phi_{n}(z)$ of degree $n(n \geqq 0)$ with positive leading coefficients such that

$$
\frac{1}{2 \pi} \int_{-\pi}^{\pi} \phi_{n}\left(e^{i \theta}\right) \phi_{m}\left(e^{-i \theta}\right) f(\theta) d \theta=\delta_{n, m} .
$$

It is true that $\left|\alpha_{n}\right|<1$ for all $n$ (see $[2, \S 4]$ ), in case $f(\theta)$ is positive, and we set

$$
k_{n}^{2}=\prod_{m=1}^{n}\left(1-\left|\alpha_{m}\right|^{2}\right)^{-1}
$$

(a) $A$ difference system. Let the polynomial $u_{n}(z)$ of at most degree $n$ in $z$ be defined by 


$$
u_{n}(z)=\frac{k_{n}}{\left(c_{0}\right)^{1 / 2}} z^{n} \bar{\phi}_{n}\left(z^{-1}\right),
$$

where $k_{n} /\left(c_{0}\right)^{1 / 2}$ is the positive square root of $k_{n}^{2} / c_{0}>0$ and where $\bar{\phi}_{n}(z)$ is the polynomial whose coefficients are the conjugates of the coefficients in $\phi_{n}(z)$. In [2] it was shown that $u_{n}(z)$ and $\alpha_{n}$ are related by the difference system

$$
u_{n}(z)-u_{n-1}(z)=\alpha_{n} z^{n} \bar{u}_{n}\left(z^{-1}\right), \quad \quad u_{0}=1 / c_{0} .
$$

In fact, since $\left|\alpha_{n}\right|<1,(2.4)$ uniquely determines $u_{n}(z)$, which in turn determines the polynomials $\phi_{n}(z)$. Thus, we have an alternative approach to polynomials orthogonal on the unit circle using (2.4) and starting with a given sequence $\left\{\alpha_{n}\right\}$ with $\left|\alpha_{n}\right|<1$. It was also shown in [2] that if $\left\{\alpha_{n}\right\}$ is given

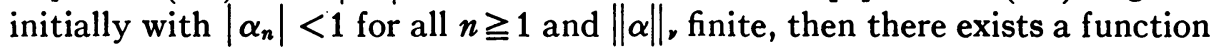
$f(\theta)>0$ with respect to which $\phi_{n}(z)$ defined by (2.3) and (2.4) are orthonormal. Theorem 1.1 now gives a more or less complete answer to the question: what convergence properties of $f(\theta)$ and of $\left\{\alpha_{n}\right\}$ are equivalent under this correspondence?

(b) Asymptotic behavior of $D_{n}(f)$. For the moment all of the functions $f(\theta)$ will be such that $\log f(\theta)$ is integrable on $-\pi \leqq \theta \leqq \pi$. We introduce the notation

$$
\begin{aligned}
\log f(\theta) & \sim \sum_{m=-\infty}^{\infty} d_{m} e^{i m \theta}, \\
G(f) & =\exp \left\{\frac{1}{2 \pi} \int_{-\pi}^{\pi} \log f(\theta) d \theta\right\} .
\end{aligned}
$$

Szegö $[3$, p. 76$]$ has proved the following theorem concerning the asymptotic behavior of $D_{n}(f)$.

Theorem (Szegö). If $f(\theta)>0$ and if $f^{\prime}(\theta)$ satisfies the Lipschitz condition

$$
\left|f^{\prime}\left(\theta_{1}\right)-f^{\prime}\left(\theta_{2}\right)\right|<K\left|\theta_{1}-\theta_{2}\right|^{\gamma}, \quad 0<\gamma \leqq 1,
$$

then there exists a finite limit

$$
\lim _{n \rightarrow \infty} \frac{D_{n}(f)}{G(f)^{n+1}}=\exp \left\{\sum_{m=1}^{\infty} m\left|d_{m}\right|^{2}\right\} .
$$

In a subsequent paper $\mathrm{Kac}$ [4] proved this theorem in the case that the Lipschitz condition is replaced by a moment condition $\sum\left|k c_{k}\right|<\infty$. In $\$ 9$, we find the exact conditions on the $\left\{\alpha_{n}\right\}$ sequence in order that $D_{n}(f) / G(f)^{n+1}$ possesses a finite limit. As a corollary we will deduce the best possible theorem of the Szegö type with a moment condition.

ThEOREM 2.1. Let $f(\theta)>0$ be continuous and let 


$$
\left.\sum_{-\infty}^{\infty}|k|\right|^{1 / 2}\left|c_{k}\right|<\infty
$$

Then, there exists a finite limit

$$
\lim _{n \rightarrow \infty} \frac{D_{n}(f)}{G(f)^{n+1}}=\exp \left\{\sum_{m=1}^{\infty} m\left|d_{m}\right|^{2}\right\} .
$$

(c) A connection with the Wiener-Lévy Theorem. The proofs given below will imply certain properties for the polynomials $\phi_{n}(z)$ which are interesting when considered in the light of the following classical Wiener-Lévy Theorem (see $[5$, p. 245]).

Theorem (WIENER-LÉvy). Let $g(\theta)$ be such that $\|g\|_{\nu}$ is finite and let $\psi(z)$ be an analytic function regular at every point of the range of $g(\theta),-\pi \leqq \theta \leqq \pi$.

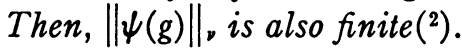

In addition to the mild similarity between the above and Corollary 1.1, there is the following fact which says the Wiener-Lévy Theorem is only loosely connected to the use of Fourier series in defining the norm (1.5).

ThEOREM 2.2. Let $f(\theta)>0$ be continuous and such that $\|f\|_{\nu}$ is finite, and let $\left\{\phi_{m}(z)\right\}$ be the Szegö polynomials associated with $f(\theta)$. Let $g(\theta)$ be an integrable function with the following expansions in terms of $e^{i k \theta}$ and $\phi_{k}\left(e^{i \theta}\right)$ :

$$
g(\theta) \sim \sum_{-\infty}^{\infty} G_{k} e^{i k \theta} \sim \sum_{-\infty}^{\infty} g_{k} \phi_{k}\left(e^{i \theta}\right)
$$

with $\phi_{-k}\left(e^{i \theta}\right)=\bar{\phi}_{k}\left(e^{-i \theta}\right)$. Then, $\sum \nu(n)\left|G_{n}\right|<\infty$ if, and only if, $\sum \nu(n)\left|g_{n}\right|<\infty$.

In particular Theorem 2.2 states that the class $Q_{\nu}$ of functions with finite norm $\|f\|_{\nu}$ can be defined in a variety of ways. It also states that the WienerLévy Theorem is valid if the norm in (1.5) is replaced by a similar sum using the coefficients in the expansion in terms of Szegö polynomials $\phi_{m}(z)$ of any sufficiently nice function $f(\theta)$.

$\S \S 3$ and 4 consist primarily of preliminaries, while $\$ \$ 5$ through 7 each contain one step of the proof of Theorem 1.1. Finally, we consider the applications in $\$ \$ 8$ and 9.

3. Properties of polynomials. In this section we summarize some properties of polynomials associated with $f(\theta)$. For details the reader is referred to [2]. We will use the notation introduced already in (1.1)-(1.6).

Every integrable function $f(\theta)$ with $D_{n}(f) \neq 0$ for $n \geqq 0$ determines uniquely (to within a plus or a minus sign) two sequences $\left\{\phi_{n}(z)\right\}$ and $\left\{\psi_{n}(z)\right\}$ of polynomials, called Szegö polynomials, such that

(2) Actually it is necessary that $\psi(z)$ be analytic at every point of the range of the function defined by the absolutely convergent Fourier series of $g(\theta)$. 
(i) $\phi_{n}(z)$ and $\psi_{n}(z)$ are polynomials of degree $n$ in $z$ and $1 / z$, respectively, with equal leading coefficients,

$$
\frac{1}{2 \pi} \int_{-\pi}^{\pi} \phi_{n}(z) \psi_{m}(z) f(\theta) d \theta=\delta_{n, m}, \quad\left(z=e^{i \theta}\right) .
$$

If $f(\theta) \geqq 0(\not \equiv 0)$, then $D_{n}(f)>0$ and $\phi_{n}(z)$ has no zeros in $|z| \leqq 1$. Moreover, the coefficient of $z^{-m}$ in $\psi_{m}(z)$. for real $f(\theta)$, is the conjugate of the coefficient of $z^{m}$ in $\phi_{m}(z)$.

For the $\alpha_{n}$ and $\beta_{n}$ of (1.3)-(1.4),

$$
k_{n}^{2} \equiv \prod_{m=1}^{n}\left(1-\alpha_{m} \beta_{m}\right)^{-1}=c_{0} \frac{D_{n-1}(f)}{D_{n}(f)},
$$

and $\alpha_{m} \beta_{m} \neq 1$ for all $m \geqq 1$. We define polynomials $u_{n}(z)$ and $v_{n}(z)$ by

$$
\begin{aligned}
& u_{n}(z)=\frac{k_{n}}{\left(c_{0}\right)^{1 / 2}} z^{n} \psi_{n}(z), \\
& v_{n}(z)=\frac{k_{n}}{\left(c_{0}\right)^{1 / 2}} z^{-n} \phi_{n}(z),
\end{aligned}
$$

where $k_{n} /\left(c_{0}\right)^{1 / 2}$ is one of the two square roots of $k_{n}^{2} / c_{0}$. The leading coefficient of $\phi_{n}(z)$ and of $\psi_{n}(z)$ is $k_{n} /\left(c_{0}\right)^{1 / 2}$. It is understood that once $k_{n} /\left(c_{0}\right)^{1 / 2}$ has been chosen as a particular one of the two square roots of $D_{n-1}(f) / D_{n}(f)$, it remains fixed throughout the discussion and is the same $k_{n} /\left(c_{0}\right)^{1 / 2}$ which appears in (3.3). This means that $u_{n}(z)$ and $v_{n}(z)$ are uniquely determined by $f(\theta)$. Moreover, for all $n \geqq 1$

$$
\begin{aligned}
& u_{n}(z)-u_{n-1}(z)=\alpha_{n} z^{n} v_{n}(z), \\
& v_{n}(z)-v_{n-1}(z)=\beta_{n} z^{-n} u_{n}(z),
\end{aligned} \quad u_{0}=v_{0}=1 / c_{0} .
$$

If $f(\theta)$ is real, $\alpha_{n}=\bar{\beta}_{n}$, the coefficient of $z^{-m}$ in $v_{n}(z)$ is the conjugate of the coefficient of $z^{m}$ in $u_{n}(z)$ and

$$
k_{n}^{2}=\prod_{m=1}^{n}\left(1-\left|\alpha_{m}\right|^{2}\right)^{-1} .
$$

If $f(\theta) \geqq 0(\not \equiv)$, then $\left|\alpha_{n}\right|<1$ for all $n \geqq 1$ and $u_{n}(z)$ has no zeros in $|z| \leqq 1$. Moreover, the function $f_{n}(\theta)=k_{n}^{2} /\left|u_{n}\left(e^{i \theta}\right)\right|{ }^{2} c_{0}$ determines according to (1.3) the sequence $\alpha_{1}, \alpha_{2}, \cdots, \alpha_{n}, 0,0,0, \cdots$.

Corresponding to the orthogonality relation (3.1), one has

$$
\begin{array}{ll}
\frac{1}{2 \pi} \int_{-\pi}^{\pi} u_{n}\left(e^{i \theta}\right) f(\theta) e^{-i m \theta} d \theta=\delta_{m 0}, & (0 \leqq m \leqq n), \\
\frac{1}{2 \pi} \int_{-\pi}^{\pi} v_{n}\left(e^{i \theta}\right) f(\theta) e^{i m \theta} d \theta=\delta_{m 0}, & (0 \leqq m \leqq n) .
\end{array}
$$


Relation (3.6) uniquely determines $u_{n}(z)$ and $v_{n}(z)$ and is equivalent to (3.1) using (3.3).

4. Normed spaces. Let $a_{\nu}$ denote the space of functions $f(\theta)$ integrable over $-\pi \leqq \theta \leqq \pi$ and such that $\|f\|_{\nu}$ as given in (1.5) is finite. Then, $a_{\nu}$ is a Banach algebra with norm $\|f\|_{\nu}$. If the Fourier coefficients of $f(\theta)$ in $a_{\text {, }}$ vanish for negative $k$, i.e. $c_{k}=0$ for $k<0$, we write $f(\theta) \in Q_{*}^{+}$. We note that $Q_{n}^{+}$is also a Banach algebra with norm $\|f\|_{\text {. }}$. Using the Wiener-Lévy Theorem, it can be shown that if $f(\theta) \in Q_{,}^{+}$and

$$
\sum_{0}^{\infty} c_{k} z^{k} \neq 0 \quad \text { in }|z| \leqq 1,
$$

then $1 / f(\theta) \in Q_{\nu}^{+}$and $\log f(\theta) \in Q_{\nu}^{+}$. We also use the space $a_{\nu}^{-}$of all functions $f(\theta) \in a_{\nu}$ whose Fourier coefficients vanish for positive $k$. Sometimes to emphasize the manner in which a function $g(\theta)$ depends on $\theta$ we will write $\|g(\theta)\|_{\nu}$.

We return for a moment to the functions $u_{n}(z)$ and $v_{n}(z)$ determined from (3.4). Let $\gamma_{k}=\max \left\{\left|\alpha_{k}\right|,\left|\beta_{k}\right|\right\}$. In terms of $\gamma_{k}$ we can find some useful norm inequalities for $u_{n}\left(e^{i \theta}\right)$ and $v_{n}\left(e^{i \theta}\right)$.

LEMMA 4.1. For every $n \geqq 0$

$$
\left\|u_{n}\left(e^{i \theta}\right)\right\|_{\nu} \leqq\left|\frac{k_{n}^{2}}{c_{0}}\right| \prod_{m=1}^{n}\left(1+\nu(m) \gamma_{m}\right),
$$

and for every $n \geqq k \geqq 0$

$$
\left\|u_{n}\left(e^{i \theta}\right)-u_{k}\left(e^{i \theta}\right)\right\|_{\nu} \leqq \sum_{m=k+1}^{n} \nu(m) \gamma_{m}\left\|v_{m}\left(e^{i \theta}\right)\right\|_{\nu} .
$$

Similar inequalities hold for $v_{n}\left(e^{i \theta}\right)$.

Proof. Relation (3.4) implies that

$$
u_{n}(z)=\frac{u_{n-1}(z)+\alpha_{n} z^{n} v_{n-1}(z)}{\left(1-\alpha_{n} \beta_{n}\right)} .
$$

Thus,

$$
\left\|u_{n}\right\|_{\nu} \leqq \frac{\left\|u_{n-1}\right\|_{\nu}+\gamma_{n} \nu(n)\left\|_{v_{n-1}}\right\|_{\nu}}{\left|1-\alpha_{n} \beta_{n}\right|}
$$

with a similar inequality for $\left\|v_{n}\right\|_{\text {. }}$ Inequality (4.2) follows by induction. To prove (4.3), we use (3.4) and write

$$
\left\|u_{n}-u_{k}\right\|_{\nu} \leqq\left\|u_{n-1}-u_{k}\right\|_{\nu}+\gamma_{n} \nu(n)\left\|v_{n}\right\|_{\nu},
$$

and the proof proceeds by induction.

Lemma 4.1 states in particular the following: 
CoROllaRy 4.1. If $\|\gamma\|_{\nu} \equiv \sum_{m=1}^{\infty} \nu(m) \gamma_{m}$ is finite, then there exist functions $U(\theta) \in Q_{\nu}^{+}$and $V(\theta) \in Q_{\nu}^{-}$so that

$$
\left\|u_{n}\left(e^{i \theta}\right)-U(\theta)\right\|_{\nu} \rightarrow 0 \text { and }\left\|v_{n}\left(e^{i \theta}\right)-V(\theta)\right\|_{\nu} \rightarrow 0 .
$$

An important property of these functions $U(\theta)$ and $V(\theta)$ was demonstrated in $[2, \S 4]$. We summarize it here in the real case for later reference.

Lемма 4.2. If $\alpha_{n}=\bar{\beta}_{n},\left|\alpha_{n}\right|<1$, and $\|\alpha\|_{\nu}$ is finite, then $u_{n}\left(e^{i \theta}\right)$ defined by (3.4) satisfies (3.6) with

$$
f(\theta)=k_{\infty}^{2} /|U(\theta)|^{2} c_{0},
$$

where $k_{\infty}^{2}=\lim k_{n}^{2}$ and where $U(\theta)$ is the limit in Corollary 4.1.

In the following sections we will have occasion to use the notation

$$
u_{n}(z)=\sum_{0}^{n} u_{n m} z^{m}, \quad v_{n}(z)=\sum_{-n}^{0} v_{n m} z^{m}
$$

5. Step one. In this section we deal with functions $f(\theta)$ of the form

(i) $f(\theta) \in Q_{\nu}$,

$$
\text { (ii) } D_{n}(f) \neq 0 \quad \text { for } n \geqq 0 \text {, }
$$$$
\text { (iii) } \log f(\theta) \in Q_{\nu} \text {. }
$$

Let $d_{k}$ be the $k$ th Fourier coefficient of $\log f(\theta)$ and let

$$
\begin{aligned}
& A(\theta)=\exp \left\{\sum_{0}^{\infty} d_{k} e^{i k \theta}\right\}, \\
& B(\theta)=\exp \left\{\sum_{-\infty}^{-1} d_{k} e^{i k \theta}\right\} .
\end{aligned}
$$

Then, $f(\theta)=A(\theta) B(\theta), A(\theta) \in Q_{\nu}^{+}$and $B(\theta) \in Q_{\nu}^{-}$. Moreover, $\hat{A}(\theta)=1 / A(\theta)$ $\in Q_{\nu}^{+}$and $\hat{B}(\theta)=1 / B(\theta) \in Q_{\nu}^{-}$

Let two functions $g(\theta)=\sum_{k=0}^{n} g_{k} e^{i k \theta}$ and $h(\theta)=\sum_{k=0}^{n} h_{k} e^{i k \theta}$ be related according to the formula

$$
\frac{1}{2 \pi} \int_{-\pi}^{\pi} h(\theta) f(\theta) e^{-i k \theta} d \theta=g_{k}, \quad 0 \leqq k \leqq n .
$$

Condition (5.1ii) insures that the relationship between $g(\theta)$ and $h(\theta)$ just indicated is unique. We now state an important lemma.

LEMMA 5.1. If $f(\theta)$ satisfies (5.1), there exists an integer $N$ and a constant $M$, both depending only on $f(\theta)$, such that for any polynomials $g(\theta)$ and $h(\theta)$ related by (5.2) 


$$
\|h\|_{\nu} \leqq M\left[\|g\|_{\nu}+\sum_{m=0}^{N} \nu(m)\left|h_{m}\right|\right] .
$$

Proof. We denote the Fourier coefficients of $A(\theta), B(\theta), \hat{A}(\theta)$, and $\hat{B}(\theta)$ by $A_{n}, B_{n}, \hat{A}_{n}$, and $\hat{B}_{n}$, respectively. Consider first

$$
h(\theta) f(\theta)=\hat{g}(\theta)=\sum_{m=-\infty}^{\infty} g_{m} e^{i m \theta}
$$

where $g_{m}$ for $m$ outside $0 \leqq m \leqq n$ is defined by (5.4). Then,

$$
h(\theta) B(\theta)=\sum_{k=-\infty}^{n} w_{k} e^{i k \theta}=\hat{g}(\theta) \hat{A}(\theta) .
$$

Calling the function in (5.5) $w(\theta)$, we get

$$
\begin{aligned}
\|w\|_{\nu} & \leqq \sum_{k=-\infty}^{n} \nu(k) \sum_{m=-\infty}^{k}\left|g_{m}\right|\left|A_{k-m}\right| \\
& \leqq\|\dot{A}\|_{\nu} \sum_{m=-\infty}^{n} \nu(m)\left|g_{m}\right| .
\end{aligned}
$$

But, if (5.5) is written in the form $h(\theta)=w(\theta) \hat{B}(\theta)$, then $\|h\|_{\nu} \leqq\|w\|_{\nu} \cdot\|\hat{B}\|_{\nu}$ so that by (5.6)

$$
\|h\|_{\nu} \leqq\|\hat{A}\|_{\nu}\|\hat{B}\|_{\nu} \sum_{k=-\infty}^{n} \nu(k)\left|g_{k}\right|
$$

From (5.4), we find

$$
g_{k}=\sum_{m=n}^{n} h_{m} c_{k-m}
$$

so that for any $N>0$

$$
\begin{aligned}
\sum_{k=-\infty}^{0} \nu(k)\left|g_{k}\right| & \leqq \sum_{m=0}^{n} \nu(m)\left|h_{m}\right| \sum_{k=-\infty}^{0} \nu(k-m)\left|c_{k-m}\right| \\
& \leqq\|f\|_{\nu} \sum_{m=0}^{N} \nu(m)\left|h_{m}\right|+\sum_{m=N+1}^{n} \nu(m)\left|h_{m}\right| \sum_{k=-\infty}^{N-1} \nu(k)\left|c_{k}\right| .
\end{aligned}
$$

Now, let $N$ be chosen so large that

$$
\|\hat{A}\|_{\nu}\|\hat{B}\|_{\nu} \sum_{k=-\infty}^{-N-1} \nu(k)\left|c_{k}\right|<1 / 2
$$

From (5.8) and (5.7) we deduce that 


$$
\begin{aligned}
\|h\|_{\nu} \leqq & \|\hat{A}\|_{\nu}\|\hat{B}\|_{\nu}\left[\sum_{k=0}^{n} \nu(k)\left|g_{k}\right|+\|f\|_{\nu} \sum_{m=0}^{N} \nu(m)\left|h_{m}\right|\right] \\
& +\frac{1}{2} \cdot \sum_{m=N+1}^{n} \nu(m)\left|h_{m}\right| .
\end{aligned}
$$

Since the second term on the right in (5.9) is less than or equal to $\|h\|_{\nu} / 2$, the proof is completed.

It is interesting to consider a simple example to show that condition (iii) in (5.1) is essential to the truth of Lemma 5.1. Let $f(\theta)=1-\lambda e^{i \theta}$. Then, the polynomials $g(\theta)=1$ and $h(\theta)=\sum_{k=0}^{n} \lambda^{k} e^{i k \theta}$ are related according to (5.2). Moreover,

$$
\|h\|_{\nu} \geqq \sum_{k=0}^{n}\left|\lambda^{k}\right|=\frac{|\lambda|^{n+1}-1}{|\lambda|-1} .
$$

If $|\lambda| \geqq 1,\|h\|$, becomes infinite as $n$ becomes infinite and an inequality like 5.3) with $\|g\|_{\nu}=\nu(0)$ is impossible. On the other hand, $|\lambda|<1$ is exactly the condition that $\log f(\theta) \in a_{\nu}$.

The functions $u_{n}(z)$ and $v_{n}(z)$ determined by $f(\theta)$ by means of (3.6) are of the type discussed in Lemma 5.1. If $d_{k}$ is (again) the Fourier coefficient of $\log f(\theta)$ and if we set

$$
\begin{aligned}
& U(\theta)=\exp \left\{-\sum_{k=0}^{\infty} d_{k} e^{i k \theta}\right\}, \\
& V(\theta)=\exp \left\{-\sum_{k=-\infty}^{0} d_{k} e^{i k \theta}\right\},
\end{aligned}
$$

then we have the first step of our proof as follows:

TheOREM 5.1. Let $f(\theta)$ satisfy (5.1) and let $\left|u_{n m}-U_{m}\right|$ and $\left|v_{n m}-V_{m}\right|$ approach zero for each fixed $m$, where $U_{m}$ and $V_{m}$ are the Fourier coefficients of $U(\theta)$ and $V(\theta)$ in (5.10). Then, $\left\|u_{n}\left(e^{i \theta}\right)-U(\theta)\right\|_{\nu} \rightarrow 0$ and $\left\|v_{n}\left(e^{i \theta}\right)-V(\theta)\right\|_{\nu} \rightarrow 0$.

Proof. It follows from (5.10) that

$$
\frac{1}{2 \pi} \int_{-\pi}^{\pi} U(\theta) f(\theta) e^{-i k \theta} d \theta=\delta_{k 0}, \quad 0 \leqq k<\infty .
$$

If we set $a_{n}(\theta)=\sum_{m=0}^{n} U_{m} e^{i m \theta}$, then from (5.11) and (3.6) for all $0 \leqq k \leqq n$

$$
\begin{aligned}
\frac{1}{2 \pi} \int_{-\pi}^{\pi}\left(u_{n}-a_{n}\right) f(\theta) e^{-i k \theta} d \theta & =\frac{1}{2 \pi} \int_{-\pi}^{\pi}\left(U-a_{n}\right) f(\theta) e^{-i k \theta} d \theta \\
& =\sum_{m=n+1}^{\infty} U_{m} c_{k-m} \\
& =g_{k}(n), \quad 0 \leqq k \leqq n .
\end{aligned}
$$


We apply Lemma 5.1 where $h=u_{n}-a_{n}$ to find

$$
\begin{aligned}
\left\|u_{n}-a_{n}\right\|_{\nu} & \leqq M\left[\|g(n)\|_{\nu}+\sum_{m=0}^{N} \nu(m)\left|u_{n m}-U_{m}\right|\right] \\
& \leqq M\left[\|f\|_{\nu}\left\|U-a_{n}\right\|_{\nu}+\sum_{m=0}^{N} \nu(m)\left|u_{n m}-U_{m}\right|\right] .
\end{aligned}
$$

In view of $(5.12)$, we can say $\left\|u_{n}\left(e^{i \theta}\right)-U(\theta)\right\|_{\nu} \rightarrow 0$. The proof for $v_{n}\left(e^{i \theta}\right)$ is similar and so the proof is completed.

6. Step two. In Corollary 4.1 it was noted that $u_{n}\left(e^{i \theta}\right)$ and $v_{n}\left(e^{i \theta}\right)$ converge in norm if $\|\gamma\|_{\nu}$ is finite. We show here that under only mild restrictions on $f(\theta)$ this condition is also necessary for the strong convergence of $u_{n}\left(e^{i \theta}\right)$ and $v_{n}\left(e^{i \theta}\right)$ to functions in $Q_{\nu}$.

Lemмa 6.1. Let $f(\theta)$ be a bounded, measurable function and let $D_{n}(f) \neq 0$ for all $n$. Moreover, let $u_{n}\left(e^{i \theta}\right)$ and $v_{n}\left(e^{i \theta}\right)$ converge uniformly in $-\pi \leqq \theta \leqq \pi$ to functions $U(\theta) \in Q_{\nu}$ and $V(\theta) \in Q_{\nu}$, respectively, where $U(\theta) \not \equiv 0$ (or $\left.V(\theta) \not \equiv 0\right)$. Then, $f(\theta) \in a_{\nu}, \log f(\theta) \in a_{\nu}$, and $\left\|u_{n}\left(e^{i \theta}\right)-U(\theta)\right\|_{\nu} \rightarrow 0,\left\|v_{n}\left(e^{i \theta}\right)-V(\theta)\right\|_{\nu} \rightarrow 0$. The functions $U(\theta)$ and $V(\theta)$ are necessarily those given in (5.10).

Proof. We start with

$$
\frac{1}{2 \pi} \int_{-\pi}^{\pi} v_{n}\left(e^{i \theta}\right) f(\theta) e^{i k \theta} d \theta=\delta_{k 0}, \quad 0 \leqq k \leqq n .
$$

Passing to the limit as $n$ becomes infinite

$$
\frac{1}{2 \pi} \int_{-\pi}^{\pi} V(\theta) f(\theta) e^{i k \theta} d \theta=\delta_{k 0}, \quad 0 \leqq k<\infty .
$$

In particular,

$$
V(\theta) f(\theta) \sim \sum_{m=0}^{\infty} a_{m} e^{i m \theta}, \quad a_{0}=1 .
$$

Since $u_{n}\left(e^{i \theta}\right)$ converges uniformly to $U(\theta)$, it follows that $U(\theta)=\sum_{m=0}^{\infty} U_{m} e^{i m \theta}$. From (6.1) and the preceding remark it follows that

$$
\begin{aligned}
\frac{1}{2 \pi} \int_{-\pi}^{\pi} U(\theta) V(\theta) f(\theta) e^{i k \theta} d \theta & =\sum_{m=0}^{\infty} U_{m} \frac{1}{2 \pi} \int_{-\pi}^{\pi} V(\theta) f(\theta) e^{i(k+m) \theta} d \theta & \\
& =U_{0} \delta_{k 0}, & 0 \leqq k<\infty .
\end{aligned}
$$

In a similar way we show that

$$
\frac{1}{2 \pi} \int_{-\pi}^{\pi} V(\theta) U(\theta) f(\theta) e^{-i k \theta} d \theta=V_{0} \delta_{k 0}, \quad 0 \leqq k<\infty .
$$


Thus, $V(\theta) U(\theta) f(\theta) \doteq U_{0}=V_{0}$. We first eliminate the possibility that $U_{0}=0$. Using the notation of $(6.2)$

$$
\begin{aligned}
\sum_{m=0}^{k} U_{m} a_{k-m} & =\frac{1}{2 \pi} \int_{-\pi}^{\pi} U(\theta) V(\theta) f(\theta) e^{-i k \theta} d \theta & & \\
& =U_{0} \delta_{k 0}, & & 0 \leqq k<\infty .
\end{aligned}
$$

Now, if $U_{0}=0$, then by (6.3), $U_{m}=0$ for all $m \geqq 0$. This contradicts our assumption $U(\theta) \not \equiv$. Thus, we have $U(\theta) V(\theta) f(\theta) \doteq U_{0} \neq 0$. Moreover, since $U(\theta)$ and $V(\theta)$ are continuous and $f(\theta)$ is bounded, we can find a bounded function $\hat{f}(\theta) \doteq f(\theta)$ such that

$$
U(\theta) V(\theta) \hat{f}(\theta)=U_{0} .
$$

It follows from (6.4) that $U(\theta) \neq 0$ and $V(\theta) \neq 0$ for all $-\pi \leqq \theta \leqq \pi$. By Wiener's theorem $\hat{f}(\theta)=U_{0} / U(\theta) V(\theta) \in a_{\nu}$. From (6.2), $V(\theta) \hat{f}(\theta) \in a_{\nu}^{+}$, and thus by (6.3)

$$
\sum_{m=0}^{\infty} U_{m} z^{m} \cdot \sum_{m=0}^{\infty} a_{m} z^{m}=U_{0} \quad \text { in }|z| \leqq 1 .
$$

According to (4.1) this means $\log U(\theta) \in Q_{\nu}^{+}$. In a similar way we show $\log V(\theta) \in a_{\nu}^{-}$. Finally, $\log f(\theta) \doteq \log U_{0}-\log U(\theta)-\log V(\theta) \in a_{\nu}$.

To finish the proof let us note that $U(\theta)$ and $V(\theta)$ in (5.10) are the unique functions in $a_{\nu}^{+}$and $a_{\nu}^{-}$, respectively, with $U_{0}=V_{0}$ and satisfying (6.4). The uniform convergence of $u_{n}\left(e^{i \theta}\right)$ to $U(\theta)$ implies convergence of each Fourier coefficient. The proof is completed with an application of Theorem 5.1.

THEOREM 6.1. Let $f(\theta)$ be bounded and measurable with $D_{n}(f) \neq 0$ for all $n$. Then, a necessary and sufficient condition that $u_{n}\left(e^{i \theta}\right)$ and $v_{n}\left(e^{i \theta}\right)$ converge in norm to functions $U(\theta) \not \equiv 0$ and $V(\theta) \not \equiv$ in $a_{\nu}$ is that $\|\gamma\|_{\nu}$ is finite. In either case, $f(\theta)$ and $\log f(\theta)$ are in $Q_{\nu}$, and $U(\theta)$ and $V(\theta)$ are given by (5.10).

Proof. We prove the sufficiency first. The existence of limits $U(\theta)$ and $V(\theta)$ follows from Corollary 4.1. The constant term of $u_{n}\left(e^{i \theta}\right)$ is $k_{n}^{2} / c_{0}$, which according to (3.2) has a finite nonzero limit if $\|\boldsymbol{\gamma}\|_{\nu}$ is finite. In the proof of Lemma 6.1 we saw that $U(\theta)$ was either identically zero or never zero. Thus, $U(\theta) \not \equiv$, etc. This proves sufficiency. According to Lemma 6.1, $f(\theta)$ and $\log f(\theta)$ are in $Q_{\nu}$ and the limits $U(\theta)$ and $V(\theta)$ are given in (5.10).

To prove the necessity, we will show $\|\alpha\|_{\nu}=\sum \nu(m)\left|\alpha_{m}\right|$ is finite. A similar proof is valid for $\|\beta\|_{\nu}=\sum \nu(m)\left|\beta_{m}\right|$, and the desired result follows. Consider (suppressing functional dependence on $\theta$ ) from (3.4)

$$
u_{n-1}=u_{n}-\alpha_{n} e^{i n \theta_{v_{n}}} .
$$

Comparing coefficients of $e^{i n \theta}$ on both sides of (6.5), we find that $u_{n n}=\alpha_{n} v_{n 0}$. Now, if $V(\theta) \equiv V$ is given in (5.10), the coefficient of $e^{i n \theta}$ on the right side of 


$$
\frac{u_{n-1}}{V}=\frac{u_{n}}{V}-\alpha_{n} e^{i n \theta} \frac{v_{n}}{V}
$$

is zero. Thus,

$$
\left\|\frac{u_{n-1}}{V}\right\|_{\nu} \leqq\left\|\frac{u_{n}}{V}\right\|_{\nu}+\left|\alpha_{n}\right| \nu(n)\left\|\frac{v_{n}}{V}\right\|_{\nu}-2 \nu(n)\left|\alpha_{n}\right|\left|\frac{v_{n 0}}{V_{0}}\right| .
$$

Summing (6.6), we find

$$
\sum_{m=0}^{n} \nu(m)\left|\alpha_{m}\right|\left[2\left|\frac{v_{m 0}}{V_{0}}\right|-\left\|\frac{v_{m}}{V}\right\|_{\nu}\right] \leqq\left\|\frac{u_{n}}{V}\right\|_{\nu} .
$$

Now, the term on the right of (6.7) has a finite limit as $n$ becomes infinite and the coefficient of $\nu(n)\left|\alpha_{n}\right|$ on the left approaches 1 . This completes the proof of Theoren 6.1.

7. Step three. We now finish the proof of Theorem 1.1 for the case of real-valued functions $f(\theta)$. First, we have a lemma which says that we may assume $f(\theta) \in a_{\nu}$ and $f(\theta) \neq 0$ without loss of generality.

LEMMA 7.1. Let $f(\theta)$ be a real, bounded and measurable function on $-\pi \leqq \theta$ $\leqq \pi$ with $D_{n}(f) \neq 0$. If $\|\alpha\|_{\nu}$ is finite or if $\|\log f(\theta)\|_{\nu}$ is finite, then $f(\theta) \in Q_{\nu}$ and $f(\theta)$ is a.e. of one sign.

Proof. In the real case, $\alpha_{n}=\bar{\beta}_{n}$, so that $\gamma_{n}=\left|\alpha_{n}\right|$. By Theorem $6.1, \log f(\theta)$ $\in Q$, under either hypothesis. Thus, $\log f(\theta)$ is equal a.e. to a continuous function, which is possible only if $f(\theta)$ is a.e. of one sign.

As remarked earlier, the proof of the general conjecture stated in the introduction is out of reach at present. However, in the real case we make use of two theorems of Szegö to finish the proof.

Proof of Theorem 1.1. According to the proof of Lemma 7.1 we have only to show that $\|\log f\|_{\nu}$ finite implies $\|\alpha\|_{\nu}$ is finite. From (3.2) and (3.5) and from Szegö $[3$, p. 44]

$$
\lim _{n \rightarrow \infty} \frac{k_{n}^{2}}{c_{0}}=\lim _{n \rightarrow \infty} \frac{D_{n-1}(f)}{D_{n}(f)}<\infty .
$$

Moreover, by (3.3) and Szegö [3, p. 51 (4)]

$$
\lim _{n \rightarrow \infty} u_{n}(z)=\lim _{n \rightarrow \infty} \frac{z^{n} \Phi_{n}\left(z^{-1}\right)}{\left(c_{0}\right)^{1 / 2}} k_{n}=\exp \left\{-\sum_{0}^{\infty} d_{k} z^{k}\right\}, \quad|z|<1,
$$

where $d_{k}$ is the Fourier coefficient of $\log f(\theta)$ and where the limit in (7.1) holds uniformly for $|z| \leqq \rho<1$. This means that for every fixed $m,\left|u_{n m}-U_{m}\right|$ $\rightarrow 0$, where $U_{m}$ is the Fourier coefficient of $U(\theta)$ in (5.10). By Theorem 5.1, 
$\left\|u_{n}\left(e^{i \theta}\right)-U(\theta)\right\|_{\nu} \rightarrow 0$. Moreover, $U_{0}=\lim u_{n 0}=\lim k_{n}^{2} / c_{0} \neq 0$. By Theorem 6.1, $\|\alpha\|_{\nu}$ is finite and Theorem 1.1 is proved.

8. Properties of polynomials. Combining Theorem 1.1 with previous results of the author [2], we deduce easily the following theorem. We use the notation of $\$ 2 \mathrm{a}$.

THEOREM 8.1. Let $\phi_{n}(z)(n \geqq 0)$ be a polynomial of degree $n$ in $z$ with positive leading coefficient. Then, $\left\{\phi_{n}(z)\right\}$ is orthonormal with respect to a positive, continuous weight function $f(\theta)$ on $-\pi \leqq \theta \leqq \pi$ with $\sum\left|c_{k}\right|<\infty$ if, and only if, there exists a sequence $\left\{\alpha_{n}\right\}$ such that $\left|\alpha_{n}\right|<1, \sum\left|\alpha_{n}\right|<\propto$ and $u_{n}(z)$ $=k_{n} z^{n} \bar{\phi}_{n}\left(z^{-1}\right) /\left(c_{0}\right)^{1 / 2}$ satisfies (2.4).

Proof. In [2] everything except the implication $\sum\left|c_{k}\right|<\infty$ implies $\sum\left|\alpha_{n}\right|<\infty$ was proved. This fact follows directly from Theorem 1.1 taking $\nu(n) \equiv 1$.

Before proving Theorem 2.2 we make an observation. If $\|\log f(\theta)\|_{\nu}$ is finite, then $\|\alpha\|_{\nu}$ is finite and according to Corollary 4.1 all terms in the inequality

$$
\max _{\theta}\left|\phi_{n}\left(e^{i \theta}\right)\right| \leqq\left\|e^{i n \theta} \bar{\phi}_{n}\left(e^{-i \theta}\right)\right\|_{\nu} \leqq\left(\frac{c_{0}}{k_{n}^{2}}\right)^{1 / 2}\left\|u_{n}\left(e^{i \theta}\right)\right\|_{\nu}
$$

are uniformly bounded in $n$. Thus, either series in (2.9) converges absolutely and uniformly if the coefficients are absolutely convergent.

Proof of Theorem 2.2. It is sufficient to consider the case

$$
g(\theta) \sim \sum_{0}^{\infty} G_{k} e^{i k \theta} \sim \sum_{0}^{\infty} g_{k} \phi_{k}\left(e^{i \theta}\right) .
$$

First, let $\sum \nu(k)\left|g_{k}\right|<\infty$. Then, the series on the right in (8.1) converges uniformly, and

$$
\begin{aligned}
G_{k} & =\frac{1}{2 \pi} \int_{-\pi}^{\pi} \sum_{m=0}^{\infty} g_{m} \phi_{m}\left(e^{i \theta}\right) e^{-i k \theta} d \theta \\
& =\sum_{m=k}^{\infty} g_{m} \phi_{m k} \\
& =\sum_{m=k}^{\infty} \hat{g}_{m} \bar{u}_{m, m-k}, \quad \hat{g}_{m}=\left(\frac{c_{0}}{k_{n}^{2}}\right)^{1 / 2} g_{m} .
\end{aligned}
$$

Thus, from (8.2)

$$
\sum_{k=0}^{\infty} \nu(k)\left|G_{k}\right| \leqq \sum_{m=0}^{\infty} \nu(m)\left|\hat{g}_{m}\right| \cdot\left\|\bar{u}_{m}\left(e^{-i \theta}\right)\right\|_{\nu}
$$

and since $\lim k_{n}^{2} \neq 0$, the desired result follows. 
Next assume $\sum \nu(k)\left|G_{k}\right|<\infty$. Then,

$$
g_{k}=\frac{1}{2 \pi} \int_{-\pi}^{\pi} g(\theta) f(\theta) \phi_{k}\left(e^{-i \theta}\right) d \theta=\sum_{m=0}^{k} H_{m} \bar{\phi}_{k m},
$$

where the $H_{m}$ in (8.3) are the Fourier coefficients of $g(\theta) f(\theta) \in Q_{\nu}$. If $\hat{g}_{m}$ $=\left(k_{m}^{2} / c_{0}\right)^{1 / 2} g_{m}$, then

$$
\sum_{k=0}^{\infty} \nu(k)\left|\hat{g}_{k}\right| \leqq \sum_{m=0}^{\infty} \nu(m)\left|H_{m}\right| \cdot \sum_{k=m}^{\infty} \nu(k-m)\left|u_{k, k-m}\right|
$$

To finish the proof we must show that

$$
a_{m}=\sum_{k=m}^{\infty} \nu(k-m)\left|u_{k, k-m}\right|
$$

is bounded in $m$. Equating coefficients of $e^{i(n-m) \theta}$ on both sides of (2.4), we find $u_{n, n-m}=u_{n-1, n-m}+\alpha_{n} \bar{u}_{n, m}$. Thus,

$$
\begin{aligned}
a_{m} & \leqq \sum_{n=m}^{\infty} \nu(n-m)\left|u_{n-1, n-m}\right|+\sum_{n=m}^{\infty} \nu(n-m)\left|\alpha_{n}\right|\left|\bar{u}_{n, m}\right| \\
& =a_{m-1}+\sum_{n=m}^{\infty} \nu(n-m)\left|\alpha_{n}\right|\left|\bar{u}_{n, m}\right| .
\end{aligned}
$$

Summing (8.4) on $m$

$$
\begin{aligned}
a_{m} & \leqq \sum_{k=0}^{\infty} \sum_{n=k}^{\infty} \nu(n-k)\left|\alpha_{n}\right|\left|\bar{u}_{n, k}\right| \\
& \leqq \sum_{n=0}^{\infty} \nu(n)\left|\alpha_{n}\right| \cdot\left\|\bar{u}_{n}\left(e^{-i \theta}\right)\right\|_{\nu} .
\end{aligned}
$$

This completes the proof of Theorem 2.2.

9. Asymptotic behavior of $D_{n}(f)$. In this section we consider the asymptotic behavior of $D_{n}(f)$ for $f(\theta) \geqq 0$ in terms of the sequence $\left\{\alpha_{n}\right\}$. This sequence seems ideally suited for this investigation in view of (3.2) and (3.5), which state

$$
D_{n}(f)=c_{0}^{n+1} \prod_{m=1}^{n}\left(1-\left|\alpha_{m}\right|^{2}\right)^{n+1-m}
$$

THEOREM 9.1. Let $f(\theta) \geqq 0$ be such that $f(\theta)$ and $\log f(\theta)$ are integrable on $-\pi \leqq \theta \leqq \pi$. Then, a necessary and sufficient condition that there exist a finite limit for $D_{n}(f) / G(f)^{n+1}$ or for $D_{n}(f) /\left[D_{n}(f) / D_{n-1}(f)\right]^{n+1}$ is that $\sum n\left|\alpha_{n}\right|^{2}$ be finite. Also 


$$
\lim _{n \rightarrow \infty} \frac{D_{n}(f)}{G(f)^{n+1}}=\prod_{m=1}^{\infty}\left(1-\left|\alpha_{m}\right|^{2}\right)^{-m}=\lim _{n \rightarrow \infty} \frac{D_{n}(f)}{\left[\frac{D_{n}(f)}{D_{n-1}(f)}\right]^{n+1}}
$$

Proof. The proof follows more or less directly from the two equalities

$$
D_{n}(f) /\left[D_{n}(f) / D_{n-1}(f)\right]^{n+1}=\prod_{m=1}^{n}\left(1-\left|\alpha_{m}\right|^{2}\right)^{-m}
$$

and

$$
D_{n}(f) / G(f)^{n+1}=\prod_{m=1}^{n}\left(1-\left|\alpha_{m}\right|^{2}\right)^{-m} / \prod_{m=n+1}^{\infty}\left(1-\left|\alpha_{m}\right|^{2}\right)^{n+1} .
$$

In (9.3) we have used the fact that $G(f)=\lim D_{n}(f) / D_{n-1}(f)$.

Before proving Theorem 2.1, we present a useful lemma.

LEMMA 9.1. If $f(\theta)$ is a positive continuous function, and if $u_{n}\left(e^{i \theta}\right)$ is associated with $f(\theta)$ according to (2.1)-(2.3), then for any $\nu(n)$ of the type in (1.5), $\left\|\log u_{n}\left(e^{i \theta}\right)\right\|_{\nu}$ is finite. Moreover, if

$$
\log u_{n}\left(e^{i \theta}\right)=\sum_{m=0}^{\infty} d_{m}^{(n)} e^{i m \theta}
$$

then

$$
\prod_{m=1}^{n}\left(1-\left|\alpha_{m}\right|^{2}\right)^{-m}=\exp \left\{\sum_{m=1}^{\infty} m\left|d_{m}^{(n)}\right|^{2}\right\} .
$$

Proof. Under the hypotheses on $f(\theta)$ it is true that $u_{n}(z)=k_{n} z^{n} \Phi_{n}\left(z^{-1}\right) /\left(c_{0}\right)^{1 / 2}$ is not zero in $|z| \leqq 1$ (see Szegö [3, p. 40]). Since, $\left\|u_{n}\left(e^{i \theta}\right)\right\|_{\nu}$ is finite, the Wiener-Lévy Theorem implies that $\left\|\log u_{n}\left(e^{i \theta}\right)\right\|_{\nu}$ is finite. Now, as remarked in $\S 3, f_{n}(\theta)=k_{n}^{2} /\left|u_{n}\left(e^{i \theta}\right)\right|^{2} c_{0}$ determines according to (1.3) the sequence $\alpha_{1}, \alpha_{2}, \cdots, \alpha_{n}, 0,0,0, \cdots$ We apply the Szegö Theorem to $f_{n}(\theta)$, which has any number of derivatives. That is,

$$
\begin{aligned}
\prod_{m=1}^{n}\left(1-\left|\alpha_{m}\right|^{2}\right)^{-m} & =\lim _{N \rightarrow \infty} \frac{D_{N}\left(f_{n}\right)}{G\left(f_{n}\right)^{N+1}} \\
& =\exp \left\{\sum_{1}^{\infty} m\left|d_{m}^{(n)}\right|^{2}\right\} .
\end{aligned}
$$

The lemma is proved.

Proof of Theorem 2.1. Taking $\nu(n)=1+|n|^{1 / 2}$, we see from Corollary 1.1 that $\sum n^{1 / 2}\left|\alpha_{n}\right|<\infty$. Thus, $\sum n\left|\alpha_{n}\right|^{2}$ is finite and (9.2) applies. Moreover, by Corollary $4.1\left\|u_{n}\left(e^{i \theta}\right)-U(\theta)\right\|_{\nu} \rightarrow 0$, where $U(\theta)$ is given in (5.10), which in 
turn implies that $\left\|\log u_{n}\left(e^{i \theta}\right)-\log U(\theta)\right\|_{\nu} \rightarrow 0$. This latter fact can equally well be written

$$
\sum_{m=0}^{\infty} m^{1 / 2}\left|d_{m}^{(n)}-d_{m}\right| \rightarrow 0 .
$$

Relation (9.6) implies that

$$
\sum_{0}^{\infty} m\left|d_{m}^{(n)}\right|^{2} \rightarrow \sum_{0}^{\infty} m\left|d_{m}\right|^{2}
$$

and the proof is completed by taking limits on both sides of (9.5).

We can show by example that Theorem 2.1 is precise in the sense that condition (2.7) cannot in general be replaced by a moment condition

$$
\sum|k| r\left|c_{k}\right|<\infty, \quad \gamma<1 / 2 .
$$

For if $\gamma<1 / 2$, we can take $N$ so large that $N(1-2 \gamma)>1$, and let

$$
\alpha_{n}=\left\{\begin{array}{cl}
\frac{1}{2 m^{N}} & \text { if } n=m^{2 N}, \\
0 & \text { otherwise. }
\end{array} \quad m=1,2, \cdots .\right.
$$

Then,

$$
\sum_{k=1}^{\infty}|k| \gamma\left|\alpha_{k}\right|=(1 / 2) \sum_{m=1}^{\infty} m^{N(2 \gamma-1)}<\infty,
$$

but

$$
\sum_{k=1}^{\infty} k\left|\alpha_{k}\right|^{2}=(1 / 4) \sum_{k=1}^{\infty} 1=\infty .
$$

According to Lemma 4.2 we can construct a function $f(\theta)=k_{\infty}^{2} /|U(\theta)|^{2} c_{0}$ which yields this particular $\left\{\alpha_{n}\right\}$ sequence according to (1.3). By Corollary 1.1 and (9.7), $\sum|k| r\left|c_{k}\right|<\infty$ for this function $f(\theta)$. On the other hand, (9.8) and Theorem 9.1 deny the existence of a finite limit for $D_{n}(f) / G(f)^{n+1}$.

Although we cannot find exact conditions on $f(\theta)$ so that $\sum n\left|\alpha_{n}\right|^{2}$ is finite, we can show the following theorem which points out one other amusing possibility.

THEOREM 9.2. Let $f(\theta)>0$ be continuous with $\sum\left|c_{k}\right|<\infty$ and let $k_{n}^{2}$ be concave, i.e., $2 k_{n}^{2} \geqq k_{n+1}^{2}+k_{n-1}^{2}$. Then,

$$
\frac{D_{n}(f)}{G(f)^{n+1}} \sim \exp \left\{\frac{1}{\pi} \iint\left|\frac{u_{n}^{\prime}(z)}{u_{n}(z)}\right|^{2} d \sigma\right\},
$$

where both sides of (9.9) have finite limits and where the integration on the right 
is with respect to two-dimensional Lebesgue measure extended over the circle $|z| \leqq 1$.

Proof. Taking $\nu(n) \equiv 1$ in Corollary 1.1 , we see that $\sum\left|\alpha_{n}\right|$ is finite. Moreover, $k_{n}^{2}-k_{n-1}^{2} \geqq k_{n+1}^{2}-k_{n}^{2}$, which means that $\left|\alpha_{n}\right|^{2} k_{n}^{2}=k_{n}^{2}-k_{n-1}^{2}$ is a decreasing sequence. Since $k_{n}^{2}$ has a finite limit, $\sum\left|\alpha_{n} k_{n}\right|$ is finite and the terms of this sequence are decreasing. This means $\left|n \alpha_{n}\right| \rightarrow 0$ as $n$ becomes infinite, so that $\sum n\left|\alpha_{n}\right|^{2}$ is finite. Theorem 9.1 insures the existence of a finite limit on the left in (9.9). To complete the proof we note that

$$
\frac{1}{\pi} \iint\left|\frac{u_{n}^{\prime}(z)}{u_{n}(z)}\right|^{2} d \sigma=\sum_{m=0}^{\infty} m\left|d_{m}^{(n)}\right|^{2},
$$

where $d_{m}^{(n)}$ is defined in (9.4). Finally, we apply (9.5).

\section{REFERENCES}

1. G. Baxter, Polynomials defined by a difference system, Bull. Amer. Math. Soc. vol. 66 (1960) pp. 187-190.

2. - On polynomials defined by a difference system, J. Math. Anal. Appl., to appear.

3. U. Grenander and G. Szegö, Toeplitz forms and their applications, Berkeley, University of California Press, 1958.

4. M. Kac, Toeplitz matrices, translation kernels and a related problem in probability theory, Duke Math. J. vol. 21 (1954) pp. 501-510.

5. A. Zygmund, Trigonometrical series, vol. I, 2d ed., Cambridge University Press, 1959.

AARHUS UNIVERSITET,

Aarhus, DenMark

UNIVERSITY OF MINNESOTA,

MinNEAPOLIS, MinNESOTA 